\title{
Status of the ATLAS Level-1 Central Trigger and Muon Barrel Trigger and First Results from Cosmic-Ray Data
}

\author{
G. Aielli*, A. Aloisio ${ }^{\dagger}$, M.G. Alviggi ${ }^{\dagger}$, S. Antonelli ${ }^{\ddagger}$, S. Ask ${ }^{\S}$, L. Bellagamba ${ }^{\ddagger}$, \\ S. Ben Ami ${ }^{\Uparrow}$, Y. Benhammou ${ }^{\|}$, D. Berge ${ }^{\S}$, M. Bianco** ${ }^{* *}$ M.G. Biglietti ${ }^{\dagger}$, D. Boscherini ${ }^{\ddagger}$, \\ S. Bressler ${ }^{\llbracket}$, A. Bruni ${ }^{\ddagger}$, G. Bruni ${ }^{\ddagger}$, S. Buda ${ }^{\dagger \dagger}$, P. Camarri*, V. Canale ${ }^{\dagger}$, \\ D. Caracinha ${ }^{\ddagger \ddagger}$, R. Cardarelli*, G. Carlino ${ }^{\dagger}$, G. Chiodini** ${ }^{* *}$ G. Ciapetti ${ }^{\mathrm{x}}$, M.R. Coluccia**, \\ S. Constantin ${ }^{\dagger \dagger}$, F. Conventi ${ }^{\dagger}$, R. DeAsmundis ${ }^{\dagger}$, M. DellaPietra ${ }^{\dagger}$, D. DellaVolpe ${ }^{\dagger}$, M. Dogaru ${ }^{\dagger \dagger}$, \\ D. De Pedis ${ }^{x}$, A. Di Girolamo ${ }^{x}$, A. DiCiaccio*, A. Di Mattia ${ }^{x}$, N. Ellis ${ }^{\S}$, E. Etzion", \\ P. Farthouat ${ }^{\S}$, C. Fukunaga $a^{\mathrm{x}}$, P. Gällnö ${ }^{\S}$, E. Gorini**, F. Grancagnolo**, P. Giusti ${ }^{\ddagger}$, \\ S. Haas ${ }^{\S}$, J. Haller ${ }^{\text {xii }}$, Y. Hasegawa ${ }^{\text {xii }}$, G. Iacobucci ${ }^{\ddagger}$, M. Ikeno $^{\text {xiv }}$, P. Iengo ${ }^{\dagger}$, \\ M. Ishino ${ }^{\text {xv }}$, H. Iwasaki ${ }^{\text {xiv }}$, V. Izzo ${ }^{\dagger}$, T. Kadosaka ${ }^{\text {xvi }}$, E. Kajomovitz ${ }^{\Uparrow}$, N. Kanaya ${ }^{\text {xvi }}$, \\ K. Kawagoe $^{\text {xvi }}$, T. Kawamoto ${ }^{\text {xv }}$, H. Kiyamura ${ }^{\text {xvi }}$, P. Klofver ${ }^{\S}$, T. Kobayashi ${ }^{\text {xv }}$, T. Kohno ${ }^{\S}$, \\ A. Krasznahorkay ${ }^{\S}$, T. Kubota ${ }^{\text {xv }}$, H. Kurashige ${ }^{\text {xvi }}$, T. Kuwabara ${ }^{\text {xv }}$, D. Lellouch ${ }^{\text {xvi }}$, L. Levinson ${ }^{\text {xvii }}$,

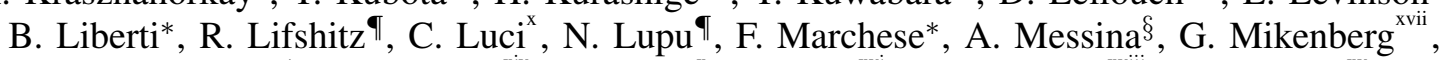 \\ A. Migliaccio ${ }^{\dagger}$, K. Nagano ${ }^{\text {xiv }}$, A. Nisati ${ }^{\mathrm{x}}$, T. Niwa ${ }^{\mathrm{xvi}}$, M. Nomachi ${ }^{\text {xviii }}$, H. Nomoto $^{\mathrm{xv}}$, \\ M. Nozaki ${ }^{\text {xiv }}$, A. Ochi ${ }^{\text {xvi }}$, C. Ohm ${ }^{\S}$, Y. Okumura ${ }^{\text {xix }}$, C. Omachi ${ }^{\text {xvi }}$, H. Oshita ${ }^{\text {xii }}$, \\ S. Patricelli ${ }^{\dagger}$, T. Pauly ${ }^{\S}$, M. Perantoni ${ }^{\mathrm{xx}}$, H. Pessoa Lima Junior ${ }^{\mathrm{xx}}$, E. Petrolo $^{\mathrm{x}}$, E. Pasqualucci ${ }^{\mathrm{x}}$, \\ F. Pastore ${ }^{\mathrm{x}}$, M. Pectu ${ }^{\dagger \dagger}$, R. Perrino** A. Polini ${ }^{\ddagger}$, M. Primavera** ${ }^{* *}$ A. Roich ${ }^{\text {xii }}$, \\ S. Rosati ${ }^{\mathrm{x}}$, A. Salamon*, H. Sakamoto ${ }^{\mathrm{xv}}$, R. Santonico*, O. Sasaki ${ }^{*}{ }^{\text {iv }}$, G. Schuler ${ }^{\S}$, \\ J.M. de Seixas ${ }^{\text {xx }}$, G. Sekhniaidze ${ }^{\dagger}$, E. Solfaroli*, S. Spagnolo**, F. Spila ${ }^{x}$, R. Spiwoks ${ }^{\S}$ \\ Y. Sugaya $^{\text {xiii }}$, T. Sugimoto ${ }^{\text {xix }}$, Y. Takahashi ${ }^{\text {xix }}$, H. Takeda ${ }^{\text {xvi }}$, T. Takeshita $^{\text {xiii }}$, S. Tanaka ${ }^{\text {xiv }}$, \\ S. Tarem M. Tomoto ${ }^{\text {xix }}$, O. Bahat Treidel ${ }^{\uparrow}$, R. Vari $^{\mathrm{x}}, \mathrm{S}$. Veneziano ${ }^{\mathrm{x}}$, T. Wengler ${ }^{\mathrm{x} i}$, \\ Y. Yamaguchi ${ }^{\text {xv }}$, Y. Yasu ${ }^{\text {xiv }}$, L. Zanello ${ }^{\mathrm{x}}$ \\ * Universita degli Studi di Roma "Tor Vergata" and INFN Roma II \\ † Universita degli Studi di Napoli "Federico II" and INFN Napoli \\ $\ddagger$ INFN Bologna and Universit degli Studi di Bologna \\ $\S$ CERN, PH Department, Switzerland \\ I Technion Israel Institute of Technology \\ $\|$ Tel Aviv University \\ ** Universita degli Studi di Lecce and INFN Lecce \\ ${ }^{\dagger}$ National Institute for Physics and Nuclear Engineering "Horia Hulubei", NIPNE-HH, Bucarest, Romania \\ $\ddagger \ddagger$ University of Lisbon, Portugal \\ ${ }^{x}$ INFN Roma and Universit di Roma La Sapienza \\ ${ }^{x i}$ Department of Physics, Tokyo Metropolitan University, Tokyo \\ xii University of Hamburg, Germany \\ xiii Department of Physics, Shinshu University, Matsumoto \\ ${ }^{\text {xiv }}$ High Energy Accelerator Research Organization (KEK), Tsukuba \\ ${ }^{x v}$ International Center for Elementary Particle Physics (ICEPP), The University of Tokyo, Tokyo \\ ${ }^{x v i}$ Department of Physics, Kobe University, Kobe \\ ${ }^{x v i i}$ Weizmann Institut of Science \\ xviii Department of Physics, Osaka University, Osaka \\ ${ }^{x i x}$ Department of Physics, Nagoya University, Nagoya \\ ${ }^{\mathrm{xx}}$ University of Rio de Janeiro, Brazil \\ ${ }^{x x i}$ University of Manchester, U.K.
}


Abstract-The ATLAS detector at CERN's Large Hadron Collider (LHC) will be exposed to proton-proton collisions from beams crossing at $40 \mathrm{MHz}$. A three-level trigger system will select potentially interesting events in order to reduce the readout rate to about $200 \mathrm{~Hz}$. The first trigger level is implemented in custom-built electronics and makes an initial fast selection based on detector data of coarse granularity. It has to reduce the rate by a factor of $10^{4}$ to less than $100 \mathrm{kHz}$. The other two consecutive trigger levels are in software and run on PC farms. We present an overview of the first-level central trigger and the muon barrel trigger system and report on the current installation status. Moreover, we show analysis results of cosmicray data recorded in situ at the ATLAS experimental site with final or close-to-final hardware.

\section{INTRODUCTION}

The event selection at the LHC is a very challenging task. At the design luminosity of $10^{34} \mathrm{~cm}^{-2} \mathrm{~s}^{-1}$ there are on average 23 collisions per bunch crossing. With beams crossing at $40 \mathrm{MHz}$, this gives an interaction rate of $1 \mathrm{GHz}$. The rate is dominated by the inelastic part of the total cross-section, which is about $70 \mathrm{mb}$. The cross-sections of many of the interesting physics processes are a factor of $10^{6}$ below that value. For example, at design luminosity the cross-section for inclusive $\mathrm{W}$ production leads to a rate of $300 \mathrm{~Hz}$, whereas the rates for some rare physics processes are much smaller. For the Standard Model Higgs boson for example with a mass of $150 \mathrm{GeV}$ the rate is expected to be in the sub-Hz region. Thus a powerful selection is needed to extract the interesting physics signals from the vast background.

The full event size of ATLAS is on the order of 1.5 MB. With $40 \mathrm{MHz}$ incoming rate, this would result in a data rate of $60 \mathrm{~TB} / \mathrm{s}$, way beyond current network and storage capabilities. Hence an online selection, that is, a trigger system, has to be set in place to reduce the incoming rate to an affordable level of $100-200 \mathrm{~Hz}$ (aiming at a data rate of $\sim 300 \mathrm{MB} / \mathrm{s}$ ) while retaining potentially interesting physics events.

ATLAS has designed a three-level trigger system which has the demanding task of finding the five in one million events that can be recorded. It aims at selecting as efficient and unbiased as possible the interesting physics events and is largely based on signatures of high-transverse-momentum particles and large missing transverse energy.

The sketch in Fig. 1 gives an overview of the design of the trigger system of ATLAS. The trigger is split in three steps. The first level (LVL1) [1] is based on coarse-resolution data from the calorimeters and dedicated fast muon detectors. It consists of the calorimeter trigger, the muon trigger, and the central trigger system. The output rate of LVL1 is required to be less than $100 \mathrm{kHz}$ with an allowed latency of $2.5 \mu \mathrm{s}$. The corresponding output data rate amounts to $\sim 150 \mathrm{~GB} / \mathrm{s}$. The remaining two trigger levels, the level-2 trigger (LVL2) and the event filter, are implemented in software and run on commercial PC farms [2]. The LVL2 trigger design is a unique feature of ATLAS. It is based on the concept of "regions of interests" (RoIs). Algorithms request full-resolution data only from a fraction of the detector, from regions that were identified by the LVL1 trigger as regions of interest. For that

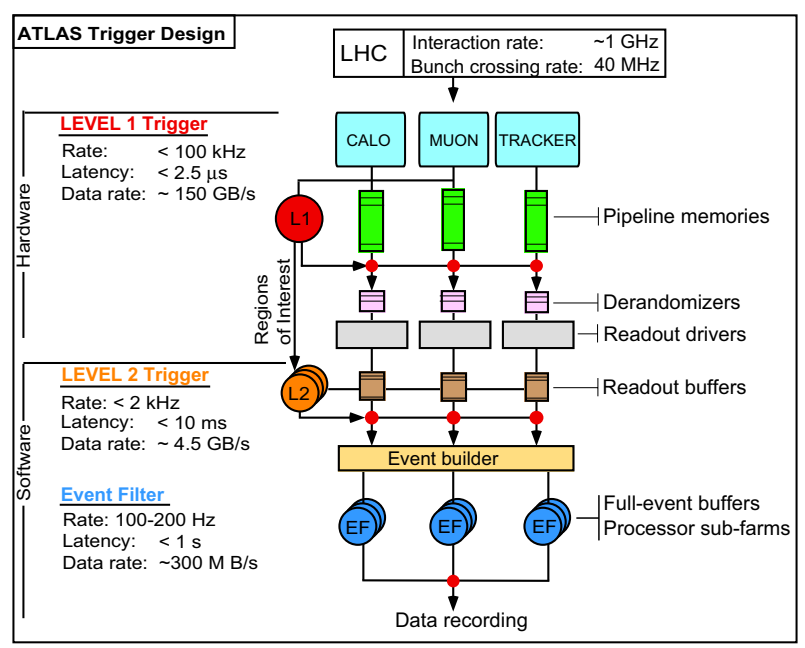

Fig. 1. The layout of the trigger system of ATLAS is shown here. There are three selection levels. The first one is in hardware, the second and third ones are done in software. In the level-1, the trigger decision is based on coarse-resolution input from muon trigger and calorimeter detectors, the data of the inner detector is only used in the higher trigger levels. The algorithms of the level-2 make use of only a fraction of the high-resolution event data, guided by regions of interest data received from the level-1 trigger. The level3 trigger algorithms refine the trigger decision once more based on the full event information.

purpose, event information like the coordinates of a particle candidate or energy and momentum values are generated by the LVL1 trigger systems and sent to the LVL2 trigger processors. Thereby the amount of full-resolution data that is accessed by the LVL2 is less than $10 \%$ of the total event size significantly reducing the processing time. At LVL2 the rate is reduced by about a factor of 50 to a few $\mathrm{kHz}$ with an allowed latency of $10 \mathrm{~ms}$. The event filter in turn has access to the full resolution data of the whole detector, it processes fully assembled events. It runs offline-like reconstruction and selection algorithms and has to provide another factor of 10 in rate reduction within a processing time of $\sim 1 \mathrm{~s}$ to arrive at the final storage rate of $100-200 \mathrm{~Hz}$.

\section{The ATLAS LVL1 TRIgGer System}

The first-level trigger system of ATLAS (cf. Fig. 2) synchronously processes information from the calorimeter and muon trigger detectors at the heartbeat of the LHC, the protonproton bunch-crossing frequency of $40.08 \mathrm{MHz}$. It comprises three sub-systems, the calorimeter trigger, the muon trigger, and the central-trigger system.

\section{A. The LVL1 Calorimeter Trigger}

The LVL1 calorimeter trigger system receives trigger signals from the calorimeter detectors, that is, the electromagnetic liquid-argon calorimeter and the hadronic scintillatortile calorimeter. On-detector electronics combine the analogue signals to 7200 trigger towers, which are passed on to the calorimeter-trigger system in the counting rooms next to the ATLAS cavern. The trigger system consists of three subsystems. The PreProcessor receives the trigger-tower signals with a typical granularity of $0.1 \times 0.1$ in $\eta$ and $\phi$. It digitises the 


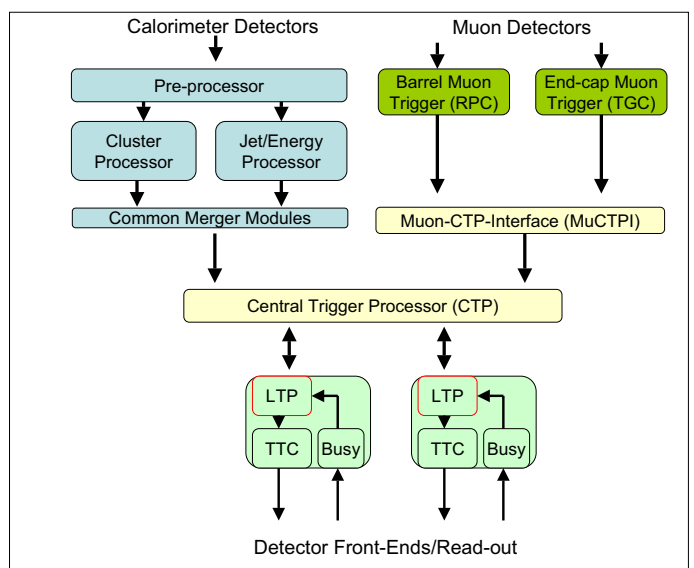

Fig. 2. Sketch of the ATLAS LVL1 trigger system. Muon and calorimeter trigger detectors provide information about event candidates to the CentralTrigger Processor (CTP), which makes the LVL1 decision. If the event is accepted, a LVL1 accept signal is fanned out to all sub-detectors to initiate the readout. The LVL1 trigger system generates (in addition to the readout data) regions of interest (RoIs) which are sent to the LVL2 trigger.

analogue signals, assigns a proton-proton bunch crossing to the trigger pulses that extend over multiple bunch crossings, and does a final lookup-table based calibration in transverse energy before sending digital data to the next two calorimeter subsystems: the algorithmic trigger processors. The Cluster Processor identifies and counts isolated electron/photon and hadron/tau lepton candidates. The transverse energy of the electron/photon (hadron/tau) candidates is discriminated against up to 16 (8) programmable thresholds. The Jet/Energysum Processor identifies jet candidates and discriminates them against 8 programmable thresholds. It also calculates missing and total transverse energy of the whole event. CommonMerger Modules carry out merging before sending the data for each of the trigger objects multiplicity and threshold information to the central-trigger system synchronously with the $40 \mathrm{MHz}$ machine. Upon reception of a LVL1-accept signal (L1A) the data are sent to the readout system and the LVL2 trigger.

The installation status of the LVL1 calorimeter trigger system of ATLAS is described elsewhere in these proceedings (see [3] and references therein).

\section{B. The LVL1 Muon Barrel Trigger}

The ATLAS muon spectrometer consists of muon chambers for precision measurements and dedicated fast muon detectors for providing information about muon candidates to the LVL1 Central Trigger. The trigger chambers are resistive-plate chambers (RPCs) in the barrel region $(|\eta|<1.05)$ and thin-gap chambers (TGCs) for the end-caps $(1.05<|\eta|<2.4)$. The trigger selects muon candidates based on transverse momentum $\left(p_{\mathrm{T}}\right)$. The trigger detectors in both the barrel and the end-caps are sub-divided in $\eta$ and $\phi$ space into trigger sectors. In total there are 64 sectors for the barrel and 144 sectors for the end-caps, the typical RoI size is approximately $\Delta \eta \times \Delta \phi=0.1 \times 0.1$.

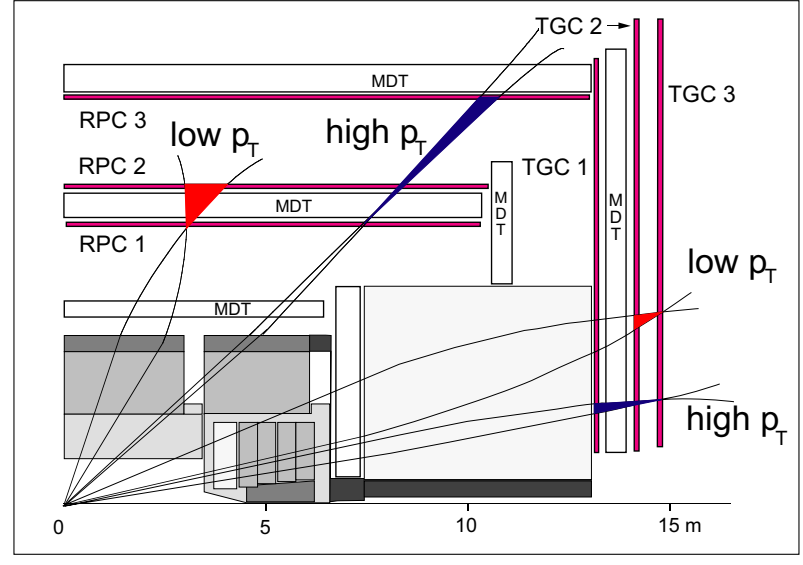

Fig. 3. Schema of the level-1 muon barrel trigger. The trigger is based on three stations. The two low- $p_{\mathrm{T}}$ inner stations, RPC1 and RPC2, are arranged on either side of the middle station of the precision chambers (MDT), at $7.8 \mathrm{~m}$ and $8.3 \mathrm{~m}$ from the interaction point. The high- $p_{\mathrm{T}}$ station RPC3 is just inside the outer MDT station at $10.2 \mathrm{~m}$ radial distance.

The RPC is a gas detector with typical space-time resolution of $1 \mathrm{~cm} \times 1 \mathrm{~ns}$ and a rate capability of $1 \mathrm{kHz} / \mathrm{cm}^{2}$. As can be seen in Fig. 3, the barrel system consists of three trigger stations arranged at different radial distances to the interaction point. The two inner stations define three low- $p_{\mathrm{T}}$ thresholds with a threshold range of approximately $6-9 \mathrm{GeV}$, the outer station is used in addition for the three high- $p_{\mathrm{T}}$ thresholds and covers a threshold range of approximately 9-35 GeV.

The on-detector trigger electronics receives as input the pattern of hits in the trigger chambers from more than $8 \times 10^{5}$ channels. The trigger algorithm starts whenever there is a hit in the middle station, RPC2, the pivot plane. It looks for a coincidence in the second station, RPC1, in a window along the line connecting the interaction point and the hit in the first station, taking the bending due to the magnetic field into account. The width of the window is programmable for up to three $p_{\mathrm{T}}$ values: the smaller the window size, the higher the cut on $p_{\mathrm{T}}$. Since each RPC station consists in fact of two RPC doublets, in both the $\eta$ and the $\phi$ projection, there are four possible coincidences. To avoid fake triggers from low-energy particles and maximize the efficiency a 3 out of 4 majority coincidence is normally required. If necessary, 2 out of 4 and 4 out of 4 coincidences can be set as well. If a low$p_{\mathrm{T}}$ coincidence is found, RPC3 is searched for a coincidence in the same way, also with three different window sizes corresponding to three $p_{\mathrm{T}}$ values. The coincidence settings for the high- $p_{\mathrm{T}}$ plane, which is also made of two RPC doublets, are normally 1 out of 2 (plus a low- $p_{\mathrm{T}}$ trigger), but a 2 out of 2 coincidence can be programmed as well if required.

The resulting muon candidate multiplicities per trigger sector, for the six $p_{\mathrm{T}}$ thresholds, are determined in offdetector electronics in the counting rooms next to the ATLAS experimental cavern. At each clock cycle, the muon trigger system sends the muon candidate multiplicities per sector for each of the six transverse-momentum thresholds to the central trigger. 


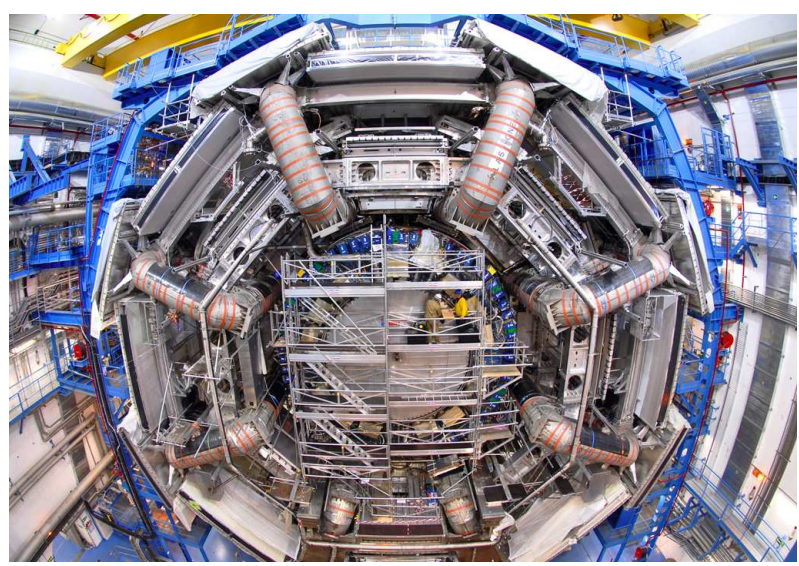

Fig. 4. A photo of the ATLAS barrel from February 2007 is shown here. The eight gray coils arranged in a circle around the centre are the barrel toroid-magnet coils. In between these one can see the (silver) chambers of the muon detector.

The installation of the LVL1 barrel muon trigger chambers is in full swing. Figure 4 shows a photo of ATLAS as of February 2007 , looking onto the front-side of the barrel. The barrel muon chambers are all installed, and so are the on-detector components of the trigger. The barrel muon spectrometer as a whole comprising the chambers, the readout electronics and the services, entered the final hardware commissioning phase in spring 2007 and will be fully operational by November $2007^{1}$. Pre-production versions of the off-detector parts of the first-level muon barrel trigger are already available and being used in integration tests. Production of the final boards commenced in spring 2007 and is expected to finish in summer 2007.

\section{The LVL1 Central Trigger}

The LVL1 Central Trigger is composed of the central-trigger processor (CTP) and the Muon-to-CTP-Interface (MuCTPI). The MuCTPI receives muon candidate information from the barrel and end-cap muon trigger chambers and resolves cases where a candidate traverses a region with overlapping trigger sectors to avoid double counting. It forms muon multiplicities for the six configurable $p_{\mathrm{T}}$ thresholds, with three bits per threshold, and sends these data to the CTP as trigger input for each bunch crossing, that is, at a frequency of $40 \mathrm{MHz}$. In case of a L1A the muon-candidate data is sent to the DAQ system at a maximum rate of $100 \mathrm{kHz}$, to be included in the ATLAS data stream, and to the LVL2 trigger as RoIs.

The CTP derives the LVL1 trigger decision based on the information received from the calorimeter and muon trigger systems and potentially input from forward and minimumbias detectors. It has a maximum of 160 trigger input bits and in addition internal trigger bits for two random triggers, two periodic triggers (that is, a trigger is generated every 1

\footnotetext{
${ }^{1}$ Note that the results presented further down are determined from data recorded with a fraction of the barrel muon detector that was equipped with temporary services and pre-production versions of the readout electronics from August 2006 to March 2007
}

out of $\mathrm{x}$ bunch crossings), and eight bunch group triggers that allow to select only certain bunch numbers to generate a trigger. The input bits are combined into 256 configurable trigger signatures, so called trigger items, each of which can be masked or pre-scaled individually. The LVL1 decision is the OR of all 256 trigger items, after application of pre-scale factors and taking busy signals from sub-detectors as well as internal dead-times into account. There are three configurable dead-times that can be applied after an L1A. One is simply a fixed number of bunch crossings during which no further trigger is generated. The other two are generated using a leakybucket algorithm which limits the number of L1As generated per time period. The number and the period are programmable.

The programmable trigger menu typically aims at selecting high-transverse-momentum leptons, photons and jets, as well as large missing and total transverse energy. In ATLAS the trigger configuration system is unified for all trigger levels and for both online data taking and offline simulation jobs. It is based on a set of relational database tables and dedicated interface software [4].

In case an event is accepted the CTP distributes the L1A signal to the various sub-detectors to initiate data readout. For these events the CTP sends its data, a GPS time stamp which corresponds to the time of the trigger decision, the 160 input bits, the internal trigger bits, and the 256 trigger bits before pre-scale factors, after pre-scale factors and after the busy or dead-time veto, to the DAQ readout system as well as to the LVL2 trigger.

The LVL1 central trigger system is already installed in the underground counting room next to the ATLAS cavern. The current MuCTPI is a demonstrator version that provides almost the full functionality of the final system, missing only some flexibility in the handling of overlaps between muon trigger sectors. Two sector input boards out of 16 in the final system are currently available, providing input for 14 out of the 208 trigger sectors each. The final boards are expected to become available for integration in the experiment mid 2007. The CTP crate is already equipped with the final boards. Both the CTP and MuCTPI are frequently used in integration and commissioning activities at the ATLAS site.

\section{ATLAS COMMISSIONING AND INTEGRATION ACTIVITIES}

Various combined cosmic-ray runs including different subdetectors of ATLAS were performed since August 2006. For some of these test runs, temporary gas systems were operational for one part (in fact 1/128) of the muon barrel detector, comprising six precision readout chambers and six RPC stations. The on-detector coincidence boards were connected to pre-production off-detector boards which determined cosmic muon candidates per $p_{\mathrm{T}}$ threshold and transmitted them to the central trigger. The central trigger in turn was configured to trigger on any non-zero number of muon candidates for any of the six thresholds and provided the L1A that initiated the readout of the sub-detectors. Figure 5 shows the $p_{\mathrm{T}}$ distribution of muon candidates from cosmic rays. To 


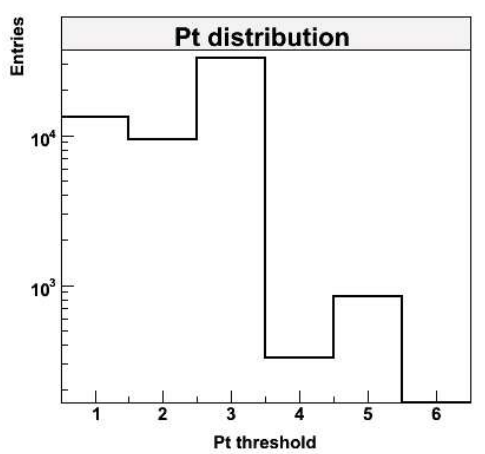

Fig. 5. Distribution of $p_{\mathrm{T}}$ from the combined cosmic runs of the muon barrel and the central trigger system. The different $p_{\mathrm{T}}$ values correspond to different coincidences fulfilled in the RPC stations.

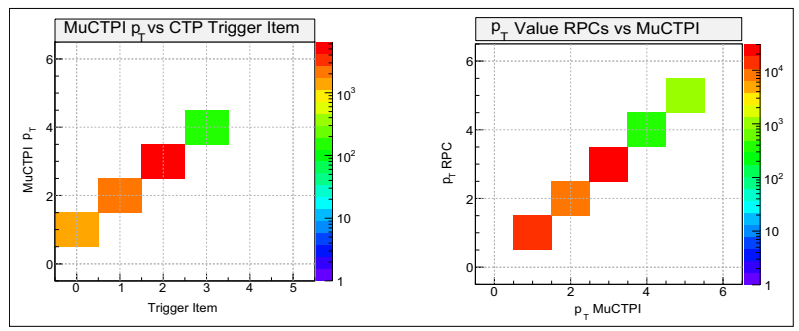

Fig. 6. Plots from the combined cosmic-ray runs of the muon-barrel detector and the central-trigger system. The left-hand side shows the correlation between the muon-candidate transverse-momentum threshold $\left(p_{T}\right)$ from the MuCTPI data and the trigger item fired in the CTP. On the right-hand side, the correlation between the muon $p_{T}$ from the RPC data and the value from the MuCTPI data is plotted.

optimise acceptance and for test purposes, the coincidence settings were loosened. A muon trigger was generated with a 2 out of 4 coincidence in the $\eta$ and $\phi$ RPC planes. The $p_{\mathrm{T}}$ thresholds correspond to different coincidences $(2 / 4,3 / 4,4 / 4$ in the low- $p_{\mathrm{T}}$ planes are thresholds $1,2,3$, accordingly in the high $-p_{\mathrm{T}}$ planes). With these loose coincidence requirements, trigger rates of $30-60 \mathrm{~Hz}$ were achieved, in agreement with simulations.

The data also allowed for validation in all involved subsystems. Figure 6 shows correlation plots of data that was transmitted from the LVL1 muon-trigger electronic to the MuCTPI, and from the MuCTPI to the CTP. On the left-hand side, we plot the trigger item fired on the CTP versus the transverse-momentum threshold at the MuCTPI, on the righthand side the candidate's momentum threshold from the RPC data versus the MuCTPI data. As desired, there is an exact one-to-one correlation in both plots demonstrating the readout and trigger data integrity.

The left-hand side of Fig. 7 shows the distribution of time differences between consecutive events, determined from the CTP time stamp. As expected, the events are exponentially distributed. The right-hand side of Fig. 7 verifies the correct functioning of the GPS-time-stamp assignment to each event. The plot shows the correlation between the difference in the bunch-crossing identifier (BCID) of consecutive events and the GPS-time difference of these events. As expected from

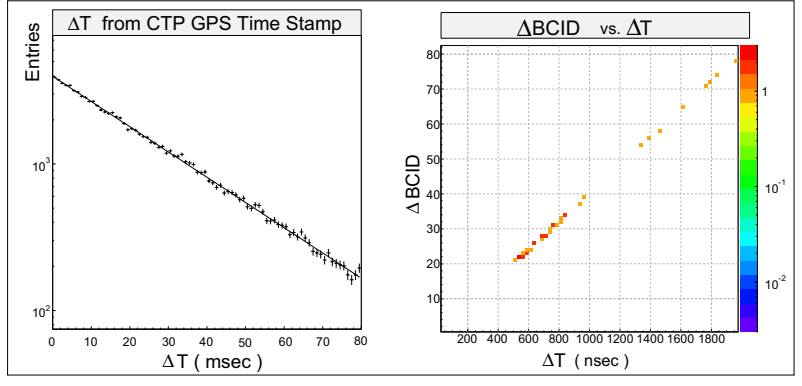

Fig. 7. Plots determined from CTP data from cosmic-ray runs. On the lefthand side the time difference between consecutive events $(\Delta \mathrm{T})$ is shown. An exponential fit (black line) describes the data very well. To validate the GPS-time-stamp assignment, we plot the correlation of the difference of the bunch-crossing identifier and the time difference for consecutive events on the right-hand side.

the fixed bunch-crossing frequency (of $40 \mathrm{MHz}$ ), there is a one-to-one correlation between the two quantities. One can also see that there are no events closer together than 20 bunch crossings, which is expected from the dead-time settings in the CTP for this particular run (for every L1A the CTP introduced a dead-time of 20 bunch crossings).

An ATLAS-wide effort to establish regular cosmic-ray data taking activities to integrate step by step the whole of ATLAS towards first collisions at the end of 2007 was started in December 2006. Cosmic rays were recorded with parts of the barrel hadronic and electromagnetic calorimeters. The CTP provided a common clock and distributed the L1As to initiate the detector readout. The second campaign in March 2007 included besides the barrel calorimeters also parts of the barrel muon detectors.

Another important milestone for the trigger system of ATLAS was reached in February 2007 with the successful integration of a full muon trigger slice up to LVL2. A LVL2 trigger algorithm was run for the first time in real online mode during a cosmic-ray run. One sector of the LVL1 muon barrel trigger was operated together with MuCTPI and CTP, which provided RoI data to the LVL2 farm. A trigger algorithm was run that selected downward-going muon candidates. The algorithm was seeded by the LVL1 RoI data and requested in turn sub-detector data from the readout system to reconstruct a muon candidate track. The algorithm was found to run as expected, reconstructing muon candidates with a mean processing time of 1 millisecond, well within the LVL2 time budget of 10 milliseconds.

\section{CONCLUSIONS}

The installation and integration of the ATLAS LVL1 trigger system is now in full swing. The final modules are mostly already produced and are being installed in the experiment as they become available. Parts of the system, especially one sector of the muon-barrel trigger and the central-trigger system, are frequently operated in test runs in combination with other ATLAS sub-detectors like the barrel calorimeters. These cosmic-ray data are used for system validation and data integrity checks. On the LVL1 trigger side, integration is 
already advanced between the central-trigger system and the muon barrel trigger. First connection tests with the calorimetertrigger system have been perforemd, and the integration with the end-cap muon trigger system is expected to commence in early summer 2007.

\section{REFERENCES}

[1] The ATLAS Collaboration, "First-level Trigger Technical Design Report", CERN/LHCC/98-14, 1998.

[2] The ATLAS Collaboration, "High-level Trigger, Data Acquisition and Controls, Technical Design Report", CERN/LHCC/2003-022, 2003.

[3] R. Achenbach et al, "Commissioning Experience with the ATLAS Level1 Calorimeter Trigger System", these proceedings.

[4] Dos Anjos et al, "The Configuration System of the ATLAS Trigger", these proceedings. 